\title{
MEASUREMENT OF TRIPLE GAUGE-BOSON COUPLINGS IN ALEPH
}

\author{
R. BRUNELIÈRE \\ LAPP Chemin de Bellevue 74940 Annecy-le-Vieux, FRANCE \\ E-mail: brunelie@lapp.in2p3.fr
}

\begin{abstract}
The triple gauge-boson couplings involving the $\mathrm{W}$ are determined using data samples collected with the ALEPH detector at mean centre-of-mass energies between $183 \mathrm{GeV}$ and $208 \mathrm{GeV}$, corresponding to an integrated luminosity of $683.6 \mathrm{pb}^{-1}$. The triple gauge-boson couplings, $\Delta g_{1}^{Z}, \Delta \kappa_{\gamma}$ and $\lambda_{\gamma}$ are measured using an optimal observable analysis of $\mathrm{W}$-pair event topology. Results from single-W and single- $\gamma$ production are included. The three couplings are measured individually assuming the two other couplings to be fixed at zero (Standard Model value). In addition, we use $\mathrm{W}$-pair events to set limits on the $\mathrm{C}$ - and P-violating couplings $g_{4}^{V}, g_{5}^{V}, \tilde{\kappa}_{V}$, and $\tilde{\lambda}_{V}$, where $\mathrm{V}$ denotes either $\gamma$ or $Z$. No deviations from the Standard Model expectations are observed.
\end{abstract}

\section{Introduction}

Triple Gauge-Boson Couplings can be separated in two main categories: vertices which involves only neutral bosons $Z$ or $\gamma$ and vertices with charged bosons $W$. The former $V Z \gamma$ and $V Z Z\left(V=Z^{*} / \gamma^{*}\right)$ are not present in the standard model at tree level. They are searched at LEP2 in the production of $Z Z$ pairs and in $Z \gamma$ events ${ }^{1}$ and won't be treated in the following note. The latter $V W^{+} W^{-}$can be found in three different physics processes at LEP2: $W W$ pair production, single $W$ production and single $\gamma$ production. The $V W^{+} W^{-}$vertices are a direct consequence of the $S U(2)_{L} \times U(1)_{Y}$ gauge theory and are present in the standard model at Born level. Their study represents a fundamental test of the non-Abelian nature of the Standard Model. The most general Lorentz invariant parametrisation of the $\gamma W^{+} W^{-}$and $Z W^{+} W^{-}$vertices can be described by 14 independent complex couplings ${ }^{2,3}$, 7 for each vertex: $g_{1}^{V}, g_{4}^{V}, g_{5}^{V}, \kappa_{V}, \lambda_{V}, \tilde{\kappa}_{V}$ and $\tilde{\lambda}_{V}$, where $V$ denotes either $\gamma$ or $Z$. Assuming electromagnetic gauge invariance, $\mathrm{C}$ - and $\mathrm{P}$-conservation, the set of 14 couplings can be reduced to 5 parameters: $g_{1}^{Z}, \kappa_{\gamma}, \kappa_{Z}, \lambda_{\gamma}$ and $\lambda_{Z}$, with Standard Model values $g_{1}^{Z}=\kappa_{Z}=\kappa_{\gamma}=1$ and $\lambda_{Z}=\lambda_{\gamma}=0$. Finally, local $S U(2)_{L} \times U(1)_{Y}$ gauge invariance introduces the constraints:

$$
\begin{gathered}
\Delta \kappa_{Z}=-\Delta \kappa_{\gamma} \tan ^{2} \theta_{W}+\Delta g_{1}^{Z}, \\
\lambda_{Z}=\lambda_{\gamma},
\end{gathered}
$$


where $\Delta$ denotes the deviation of the respective quantity from its non-zero Standard Model value, and $\theta_{W}$ is the weak mixing angle. Hence, only three parameters remain: $\Delta g_{1}^{Z}, \Delta \kappa_{\gamma}$, and $\lambda_{\gamma}{ }^{3}$. This paper presents preliminary results on the three couplings $\Delta g_{1}^{Z}, \Delta \kappa_{\gamma}$, and $\lambda_{\gamma}$ using all ALEPH data, but also updates the results from single-parameter fits to the unconstrained real and imaginary parts of the $8 \mathrm{C}$ - or P-violating couplings ${ }^{4}$.

\section{The ALEPH Detector}

A detailed description of the ALEPH detector may be found in ${ }^{5,6}$. The central part of the ALEPH detector is dedicated to the reconstruction of the trajectories of charged particles. Following a charged particle from the interaction point outwards, the trajectory is measured by a two-layer silicon strip vertex detector (VDET), a cylindrical drift chamber (ITC) and a large time projection chamber (TPC). They are immersed in a $1.5 \mathrm{~T}$ axial field and combined, they measure charged tracks with a momentum resolution of $\delta p_{T} / p_{T}=6 \times 10^{-4} p_{T} \oplus 0.005$. Photons and electrons are identified in the electromagnetic calorimeter (ECAL), situated between the TPC and the coil. It yields a relative energy resolution of $0.18 / \sqrt{E} \oplus 0.009$. The iron return yoke is equipped of 23 layers of streamer tubes and forms the hadron calorimeter (HCAL). Combined with ECAL it provides a relative energy resolution of charged and neutral hadrons of $0.85 / \sqrt{E}$. Muons are distinguished from hadrons by their distinct pattern in HCAL and by the muon chambers outside the HCAL.

\section{Event selection and kinematic reconstruction}

The selection of $W^{+} W^{-}$candidates is highly dependent of the event topology. Selected events are exclusively classified with the following order of priority: $e \nu q \bar{q}, \mu \nu q \bar{q}, \tau \nu q \bar{q}, q \bar{q} q \bar{q}$, and $l \nu l \nu$ according to the way each $W$ is decaying ${ }^{7}$.

\section{$3.1 W^{+} W^{-} \rightarrow e \nu q \bar{q}$ and $W^{+} W^{-} \rightarrow \mu \nu q \bar{q}$ events}

Semileptonic events are selected such that they contain a high energy lepton candidate and two jets. The selection of the lepton is using track isolation. The DURHAM-PE clustering algorithm is then applied to all objects that are not used to construct the lepton four-momentum, and these are forced in two jets. After this preselection, the probability for the event being signal is determined using a Neural Networks based on the momentum of the lepton, the total missing transverse momentum and the lepton isolation. To improve

tgc aleph: submitted to World Scientific on April 26, 2002 
the resolution on the reconstructed four-momenta of the $\mathrm{W}$ decay products, the events are subjected to a kinematic fit. Typical efficiency of semileptonic channels is roughly $80 \%$ for a purity of $95 \%$.

\section{2 $W^{+} W^{-} \rightarrow \tau \nu q \bar{q}$ events}

Selection of semileptonic events with a $\tau$ is slightly different from the previous case, since $\tau$ lepton can decay into hadrons. In that case, an iterative method is applied to search for the most isolated jet amongst the jets resulting from a low $y_{\text {cut }}$ value of $0.75 \mathrm{GeV} / \mathrm{c}^{2}$ with the JADE algorithm. To improve the resolution of the angular observables a kinematic fit is performed where the direction of the $\tau$ is approximated by its visible decay products. Efficiency of $\tau \nu q \bar{q}$ selection is about $54 \%$ and purity is roughly $76 \%$.

\section{3 $W^{+} W^{-} \rightarrow q \bar{q} q \bar{q}$ events}

Fully hadronic events are identified by having four separated jets contained in the detector. To extract the hadronic $W^{+} W^{-}$signal with high purity $(\simeq 85 \%)$ and efficiency $(\simeq 80 \%)$, the selection is based on the output of a neural network based on 14 variables ${ }^{7}$. Moreover, for the hadronic $W^{+} W^{-}$ events the reconstruction of the relevant information is more complicated since there is no clean signature of the $W^{-}$direction nor any information of the particle flavors in either $\mathrm{W}$ systems. In this case the four jets can be paired in three different ways. To select the best pairing with a purity of $75 \%$, a 6 -constraint kinematic fit is applied to all three possible pairings. To assign a jet pair to the $W^{+}$or $W^{-}$, a jet charge algorithm is used. The jet charge is obtained from the pseudorapidity-weighted charge for the particles forming a jet.

\section{$3.4 W^{+} W^{-} \rightarrow l \nu l \nu$ events}

The efficient of the selection of purely leptonic events is low $27 \%$, but with a high purity $97 \%$. Selection is based on missing transverse momentum, missing mass and kinematic properties of the lepton candidates. For purely leptonic $W^{+} W^{-}$events the momenta of the two neutrinos are unknown. However in the absence of Initial State Radiation and neglecting the $\mathrm{W}$ width, the constraint that the two $l \nu$ systems should have the $\mathrm{W}$ mass in combination with the usual four-momentum conservation allows a reconstruction of the neutrino momenta.

tgc aleph: submitted to World Scientific on April 26, 2002 


\section{Determination of the TGCs}

\subsection{Coupling extraction}

All the information on the couplings is contained in a five angles distribution and the electric charge of the fermions ${ }^{3}$. Since triple gauge-boson couplings contribute only linearly to the amplitude of the considered processes, the differential cross section can be expanded in these couplings $g_{i}$ as a second order polynomial

$$
\frac{d \sigma}{d \Omega}=S_{0}(\Omega)+\sum_{i} S_{1, i}(\Omega) g_{i}+\sum_{i j} S_{2, i j}(\Omega) g_{i} g_{j} .
$$

Couplings estimators are then extracted with an optimal observable method ${ }^{8}$. The general idea of optimal observables is to extract the couplings $g_{i}$ by measuring the mean values $\overline{O O}$ of distributions of suitably defined observables $O O$. In addition to the total number of selected events, the two optimal observables builded are

$$
O O_{1, i}(\Omega)=\frac{S_{1, i}(\Omega)}{S_{0}(\Omega)} \text { and } O O_{2, i j}(\Omega)=\frac{S_{2, i j}(\Omega)}{S_{0}(\Omega)} .
$$

With only Gaussian distributed variables, likelihood function is then equivalent to a least square method. In particular, including systematic errors is easy.

\subsection{Systematic uncertainties}

A complete list of all the systematics errors computed can be found in ${ }^{4}$. The main sources are coming from uncertainties on the Monte Carlo model used to generate the $W^{+} W^{-}$signal. They were estimated as the effect of higher order correction for these results, but a reduction of the error size is forseen for the final results ${ }^{9}$. The other main systematic uncertainties are due to fragmentation or final state interactions not perfectly modeled, i.e. Color Reconnection and Bose-Einstein effects.

\section{Results and conclusion}

The triple gauge-boson couplings have been measured using $\mathrm{W}$-pair events at all LEP2 energies. Combining with the ALEPH measurement from single-W production and single- $\gamma$ production, the three couplings $\Delta g_{1}^{Z}, \Delta \kappa_{\gamma}$ and $\lambda_{\gamma}$ have been measured individually. The results are

$$
\Delta g_{1}^{Z}=0.015_{-0.032}^{+0.035}
$$

tgc aleph: submitted to World Scientific on April 26, 2002 


$$
\begin{gathered}
\Delta \kappa_{\gamma}=-0.020_{-0.072}^{+0.078} \\
\lambda_{\gamma}=-0.001_{-0.031}^{+0.034},
\end{gathered}
$$

where the error includes systematic uncertainties. The corresponding $95 \%$ confidence level limits,

$$
\begin{gathered}
-0.048<\Delta g_{1}^{Z}<0.080 \\
-0.164<\Delta \kappa_{\gamma}<0.132 \\
-0.059<\lambda_{\gamma}<0.065,
\end{gathered}
$$

are in good agreement with the Standard Model expectation.

In addition, $\mathrm{W}$-pair events were used to set limits on the $\mathrm{C}$ - or P-violating couplings $g_{4}^{V}, g_{5}^{V}, \tilde{\kappa}_{V}$ and $\tilde{\lambda}_{V}$, where $V$ denotes either $\gamma$ or $Z$. Results are shown on Table 1.

Table 1. Combined results for the real and imaginary parts of the C- or P- violating couplings from W-pair production at $183-208 \mathrm{GeV}$. Each coupling is determined setting all other couplings to their Standard Model value. The error includes systematic uncertainties. The corresponding $95 \%$ confidence intervals are listed in the last column.

\begin{tabular}{|l||r|c|}
\hline Coupling & \multicolumn{1}{|c|}{ Fit result } & $95 \%$ confidence limits \\
\hline $\operatorname{Re}\left(\tilde{\kappa}_{\gamma}\right)$ & $-0.027_{-0.111}^{+0.114}$ & {$[-0.236,0.191]$} \\
\hline $\operatorname{Re}\left(\tilde{\lambda}_{\gamma}\right)$ & $0.001_{-0.090}^{+0.090}$ & {$[-0.170,0.173]$} \\
\hline $\operatorname{Re}\left(\tilde{\kappa}_{Z}\right)$ & $-0.006_{-0.060}^{+0.060}$ & {$[-0.123,0.111]$} \\
\hline $\operatorname{Re}\left(\tilde{\lambda}_{Z}\right)$ & $-0.004_{-0.048}^{+0.048}$ & {$[-0.096,0.089]$} \\
\hline $\operatorname{Re}\left(g_{4}^{\gamma}\right)$ & $0.116_{-0.158}^{+0.156}$ & {$[-0.193,0.418]$} \\
\hline $\operatorname{Re}\left(g_{5}^{\gamma}\right)$ & $-0.186_{-0.189}^{+0.188}$ & {$[-0.557,0.181]$} \\
\hline $\operatorname{Re}\left(g_{4}^{Z}\right)$ & $0.103_{-0.120}^{+0.119}$ & {$[-0.134,0.334]$} \\
\hline $\operatorname{Re}\left(g_{5}^{Z}\right)$ & $-0.130_{-0.138}^{+0.138}$ & {$[-0.400,0.141]$} \\
\hline $\operatorname{Im}\left(\tilde{\kappa}_{\gamma}\right)$ & $-0.022_{-0.057}^{+0.057}$ & {$[-0.134,0.090]$} \\
\hline $\operatorname{Im}\left(\tilde{\lambda}_{\gamma}\right)$ & $0.037_{-0.046}^{+0.045}$ & {$[-0.053,0.125]$} \\
\hline $\operatorname{Im}\left(\tilde{\kappa}_{Z}\right)$ & $-0.045_{-0.037}^{+0.037}$ & {$[-0.116,0.027]$} \\
\hline $\operatorname{Im}\left(\tilde{\lambda}_{Z}\right)$ & $0.043_{-0.029}^{+0.029}$ & {$[-0.015,0.100]$} \\
\hline $\operatorname{Im}\left(g_{4}^{\gamma}\right)$ & $0.286_{-0.132}^{+0.130}$ & {$[0.027,0.539]$} \\
\hline $\operatorname{Im}\left(g_{5}^{\gamma}\right)$ & $-0.180_{-0.216}^{+0.219}$ & {$[-0.600,0.251]$} \\
\hline $\operatorname{Im}\left(g_{4}^{Z}\right)$ & $0.167_{-0.082}^{+0.082}$ & {$[0.005,0.326]$} \\
\hline $\operatorname{Im}\left(g_{5}^{Z}\right)$ & $-0.089_{-0.142}^{+0.142}$ & {$[-0.366,0.190]$} \\
\hline
\end{tabular}




\section{References}

1. ALEPH Collaboration, Limits on anomalous neutral gauge couplings using data from $Z Z$ and $Z \gamma$ production between 183-208 GeV, contributed paper for LP01 and EPS HEP 2001, ALEPH 2001/061, CONF 2000/041.

2. K. Hagiwara, R. D. Peccei, D. Zeppenfeld and K. Hikasa, Nucl. Phys. B 282, 253 (1987).

3. G. Gounaris, J.-L. Kneur and D. Zeppenfeld, from Physics at LEP2, CERN 96-01 p. 525, editors G. Altarelli, T. Sjöstrand and F. Zwirner.

4. ALEPH Collaboration, Measurement of Triple Gauge-Boson Couplings in $e^{+} e^{-}$collisions up to $208 \mathrm{GeV}$, contributed paper for LP01 and EPS HEP 2001, ALEPH 2001/060, CONF 2000/040.

5. ALEPH Collaboration, ALEPH: A detector for Electron-Positron Annihilations at LEP, Nucl. Instrum. Methods A 294, 121 (1990).

6. ALEPH Collaboration, Performance of the ALEPH detector at LEP, Nucl. Instrum. Methods A 360, 481 (1995).

7. ALEPH Collaboration, Measurement of W-pair production and $W$ branching ratios in $e^{+} e^{-}$collisions up to $208 \mathrm{GeV}$, contributed paper to the 2001 Winter Conferences, ALEPH 2001-013, CONF 2001-010.

8. M. Diehl and O. Nachtmann, Z. Phys. C 62, 397 (1994).

9. R. Brunelière et al, hep-ph/0201304, CERN-TH-2001-274. 